\title{
HERPES ZOSTER ENCEPHALO-MYELITIS IN AN IMMUNOCOMPETENT 8 YEAR-OLD GIRL WITH DOCUMENTED VARICELLA DURING INFANCY
}

Dr Anna Stanzelova, Dr Leah Halpenny, Dr Samar Al Muntaser, Dr Bryan Lynch

\section{Introduction:}

Varicella Zoster virus (VZV) is an alpha-herpesvirus, that post primary infection becomes latent within the neurons of the dorsal roots and the cranial ganglia. It is known to be associated with neurologic complications in children, such as febrile convulsions, encephalitis, stroke or sinus thrombosis. Since the introduction of VZV PCR testing into wide practice, it has become one of the most commonly implicated viruses in CNS infection in the developed world, second only to Herpes Simplex Virus (HSV).

Herpes Zoster $(H Z)$ is a reactivation of $V Z V$ in a person with previous acute $V Z V$ infection or vaccinated with life attenuated vaccine. While in adults, $\mathrm{HZ}$ encephalitis is a recognized complication, it is rarely described in immunocompetent children.

\section{Case:}

A previously well 8 year old girl presented with a week history of painful vesicular skin eruption in left C2-C4 distribution. Two days, she developed progressive ipsilateral hemiplegia and hemi-sensory syndrome. CT showed an area of hypodensity in the contralateral thalamus. A stroke was initially suspected. Lumbar Puncture was performed which showed lymphocytosis, no organisms and normal protein and glucose. Her MRI is described below Diagnosis of Herpes zoster was made.

Treatment comprised high-dose acyclovir for 2 weeks, 5 days of intravenous immunoglobulin and 4 weeks of steroids.

Within 1 month of commencing treatment the patient regained normal function

\section{Discussion:}

Herpes Zoster $(\mathrm{HZ})$ encephalitis is a rare complication of VZV reactivation in adults. It is exceedingly rare in children. It is defined as encephalopathic state, clinical features of herpes zoster with or without virological confirmation, with or without neurologic imaging changes and inflammatory changes in CSF (Wetzel, 2002). The incidence of $\mathrm{HZ}$ encephalitis is unknown and most literature is formed by isolated case reports. Risk factors include impaired immune status, early and mild primary infection during infancy and craniocervical distribution of Zoster rash (Sang-Hoon Kim, 2017).. While the CSF in our patient was negative for VZV PCR, between a third and a half of CNS reactivations of VZV occur in the absence of a rash (Bergstrom, 1996). Common clinical presentations include altered mental status and focal neurological signs, with seizures being less frequent (Granerod, 2010). Diagnosis is often clinical, and VZV PCR may be negative, in which case intrathecal VZV antibodies and raised serum/CSF ratio can be used (Koksiniemi, 2002, Gregorio, 2006) Acyclovir is the mainstay of treatment, IV acyclovir being preferred due to poor bioavailability and CSF penetration of oral acyclovir (Gilden, 2004). Outcomes vary between minor and significant in the long-term follow up studies (Hokkanen, 1997, Wetzel, 2002).

In jurisdictions where universal vaccination for varicella has been introduced, some have reported a decrease in the incidence of acute CNS complications in children (Streng, 2017). However, mass introduction of VZV vaccine remains controversial, because of feared increase of the age and severity of primary varicella and of the incidence of Herpes Zoster (Poletti, 2013).
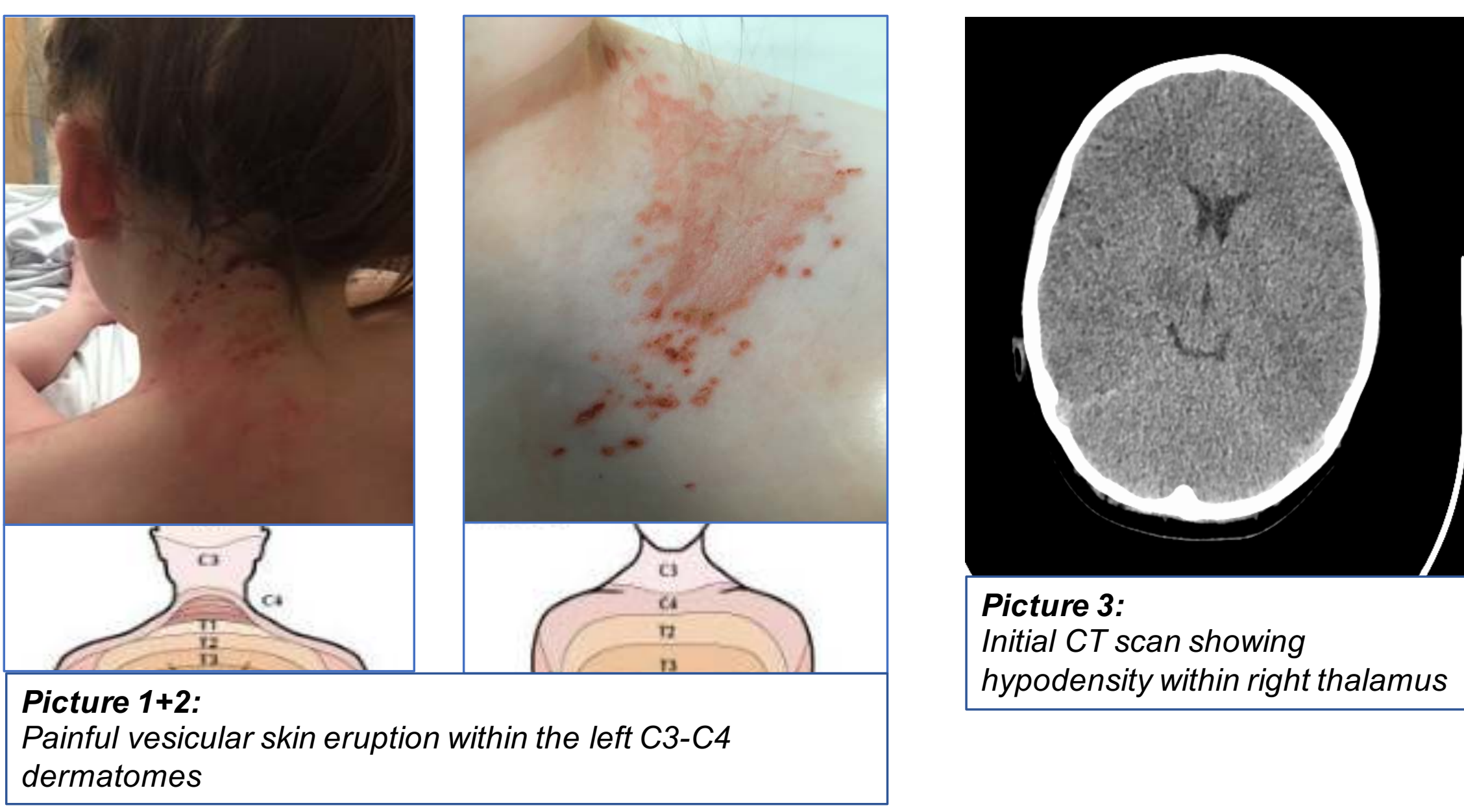

Picture 3:

Initial CT scan showing hypodensity within right thalamus

\section{Picture 4: \\ Axial T2 flair showing lesion within right thalamus and} bilateral lesions within pons

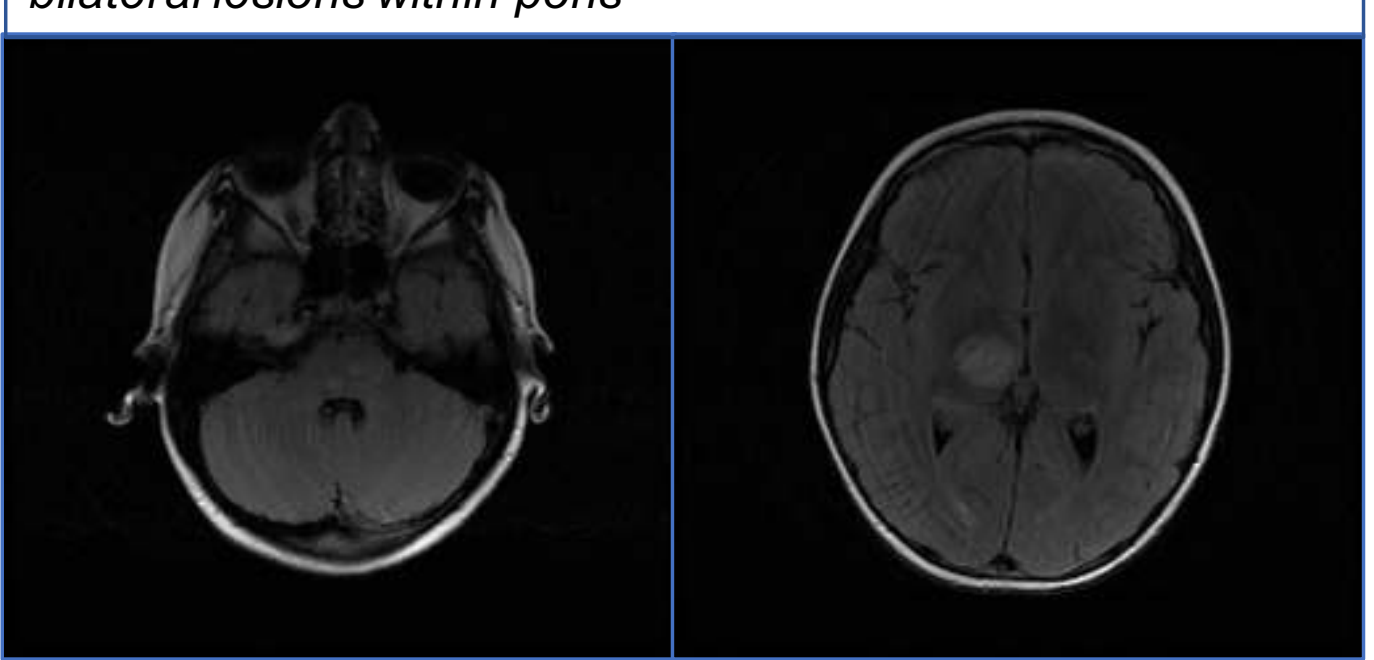

\section{Picture 5:}

Sagittal T2 flair showing C2-C4 lesion within cervical spine

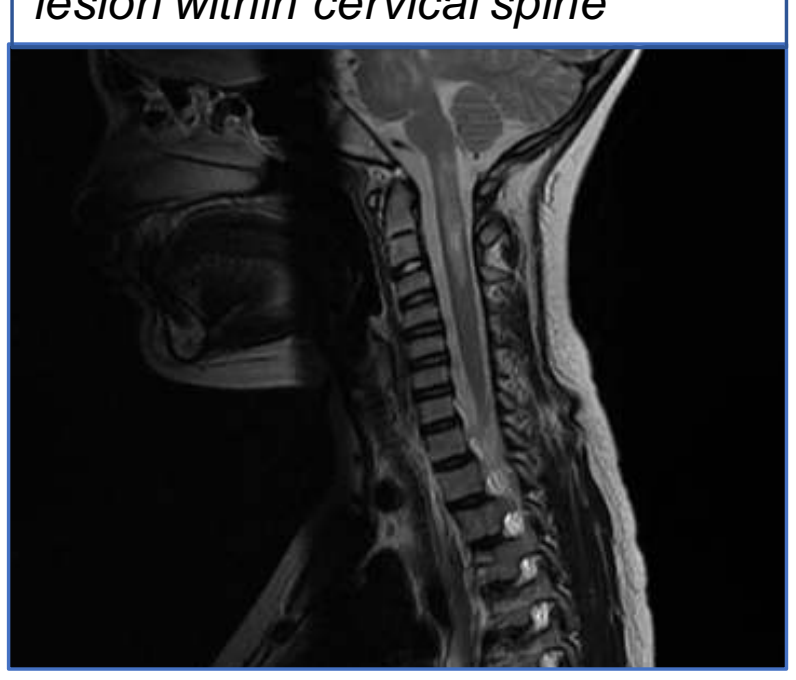

Addendum

After submission of this abstract our patient represented with new onset of painful vesicular rash and intractable hiccuping and vomiting.

Repeat MRI showed new lesions within the white matter of left hemisphere, in the area prostrema and extension of the spinal lesion. Her LP showed lymphocytosis and negative VZV PCR. While recurrent herpes Zoster encephalo-myelitis stays within the differential, she is now under investigation for other differentials including neuromyelitis optica and multiple sclerosis, the results of which are pending at time of presentation 